\title{
REMOTE SENSING AND GEOGRAPHIC INFORMATION SYSTEM BASED ROUTE PLANNING
}

\author{
Priyanka Deshmukh ${ }^{1}$, Dr. P. Y. Pawade ${ }^{2}$ and Dr. Rajashree Bothale ${ }^{3}$ \\ ${ }^{1}$ Department of Transportation Engineering, Nagpur, Maharashtra \\ ${ }^{2}$ Head of Civil Department, \\ Department of Transportation Engineering, Nagpur, Maharashtra \\ ${ }^{3}$ General Manager, Outreach Facility, \\ Indian Space Research Organization, Balanagar, Hyderabad
}

\begin{abstract}
With the progression of innovation and present day science, individuals are expecting data about the area of any protest for following purposes. GPS is a framework which has been executed and is open with no confinement. Having the office of GPS, we require a GPS gadget to compute the area from the data taken from GPS. This paper exhibits an examination on Area Based Services and is coordinated on finding and following of Android gadgets. In this usage I am utilizing Global Positioning System (GPS) and Web Services, and android advanced mobile phone gadget Bhuvan Geotag for Route Planning since Android gadgets have a worked in highlight of GPS benefit. Some most recent requesting devices and innovation utilized for this execution are Java, AVD, WAMP etc. A Location-Based Service (LBS) gives data and administration of the topographical position of the cell phone. This should be possible through the portable system and using the capacity to influence utilization of that data. So as to decide the area of client's cell phone in a LBS application, situating advances or a situating part. For example, satellite situating, cell organize situating, WLAN stations or radio signals is required. Subsequently the greater part of the present LBS administrations don't require clients to enter area physically, for example, giving road name or postal district. Specialist co-ops are likewise required since they offer unique sorts of LBS administrations to clients and are in charge of preparing administration asks for and reacts back by sending ask for comes about. Servers can compute areas or position, scan for a way or seek particular data with respect to use position. Typically all the data asked for by clients are not store and keep up by the Service suppliers, geographic information are gather and put away by content supplier information. For example, Location based related information are additionally gather and store by the content supplier. Administration servers will demands and process these information and after that came back to clients.
\end{abstract}

\section{KEYWORDS}

Location Based Service, Global Positioning System (GPS), route planning

DOI : 10.5121/civej.2018.5101 


\section{INTRODUCTION}

With evolving times, the versatile innovation has changed a considerable measure and over the most recent couple of years we have seen the landing of different new type of devices as Smartphone, camera-telephone, Android and tablet telephones. Actually, the handset business has abandoned straight forward spending handsets to ultra-present day top of the line cell phones. The present gadget is nearly everything - it is in vogue, inventive, engaging, high-performing, solid, up-to-date and multi-entrusting. Most recent contraptions can be utilized for different purposes like perusing portable, web, playing amusements, messaging, and blogging, informing, GPS, YouTube, Google look, Gmail and then some. Along this, there has been a blasting business sector for the mixed media cell phones. Present day contraptions are accompanying worked in cameras with cell phone applications, supposed applications; today are all the more searching for data on the go. This is one region of cell phone innovation upgrade that enables designers and software engineers to offer clients just what they look for under their favoured zone of intrigue. Google's Android is one of the most recent and special developments, which in a split second has assumed control over the portable market. There are 17,000 area construct fly out applications with respect to the market, and 160 million application good gadgets are possessed around the world - iPhones, Androids, Blackberries and tablet gadgets, for example- the iPad and Motorola. There are applications that can make our voyaging somewhat less demanding, more fun and more important. They let you do anything you can do on the web or with a manual, yet more rapidly and effortlessly and keeping in mind that you're progressing - with maps and GPS to reveal to you where you are and catch awesome recollections. This examination depends on improvement of an easy to use Android-based application called Vehicle Tracker.

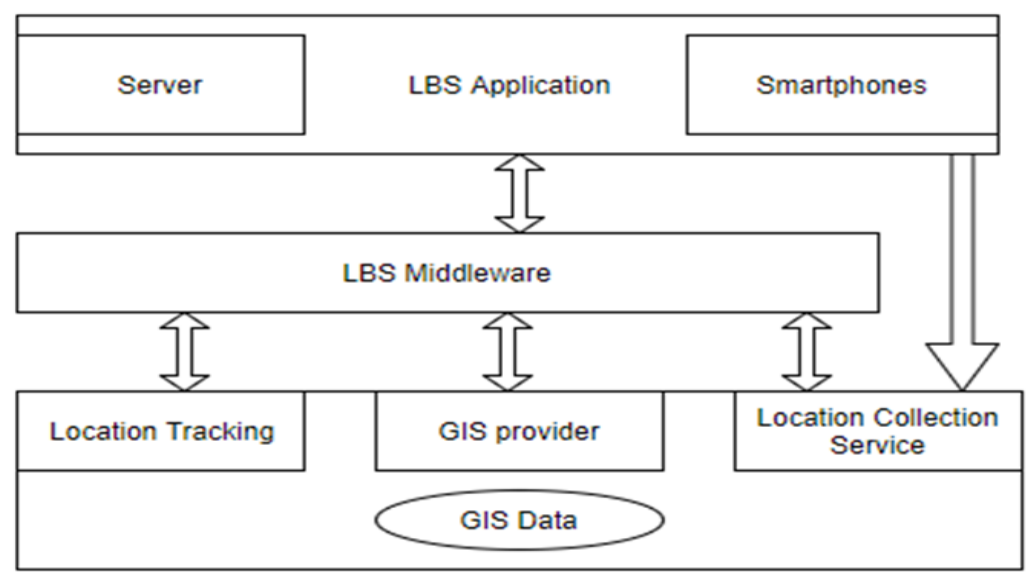

Vehicle Tracking Solutions or location tracking solution is a main supplier of GPS administration has presented a free downloadable "application" for the Android telephone that communicates with the director's Vehicle Tracking Solutions account Called Silent Traveler. This GPS following Android application offers adaptability and versatility operations administration empowering them to alter settings, get reports all from their advanced mobile phone. We will utilize GPS for finding the position of vehicle. We can track vehicles or track the person location point through android application utilizing GPS to discover a transport vehicle is utilizing a web application which requires login of Vehicle User details. We utilize the Vehicle points of interest From Vehicle Registration Form i.e. (Vehicle User Name and Vehicle user Mobile No.). This is 
Civil Engineering and Urban Planning: An International Journal (CiVEJ) Vol.5, No.1, March 2018

the Administrative Activity Form that detail we can track the area of Vehicle, just enlisted vehicles area can track.

\section{LITERATURE REVIEW}

Bus arrival and departure information is broadly handy specifically provided via the nearby public bus offerings. Using a sophisticated GPS technology, bus companies are capable of track their buses and permit customers realize the expected time of arrival Users. These days are relying more on the information, however the comfort is handiest available for public bus offerings within the town. Benjamin Y. O. Low designed a bus arrival information gadget by took gain of the traits of the carrier routes in rural regions and trip bus provider in educational institutions. Instead of depending solely on GPS facts and complicated computational algorithms to calculate the expected time of arrival of the bus, this machine used RFID to replace the ultramodern bus stop location that allowed customers to have a top level view of the real time bus vicinity. GPS become used to provide the actual-time place of the shifting bus, thanks to the Google Map. The evolved device supported participatory contributions in which the system depended on the customers to replace any unpredictable on-avenue troubles and percentage the statistics to others. A net application (Web App) was designed with interactive interface as a platform to get right of entry to all the statistics. The advanced device became examined in UTHM campus- Parit Raja town go back and forth bus. This new facility is expected to noticeably encouraged college students to apply public transport and improve their journeying reports. Through this, an arrival records system that consisted of 3 principal additives turned into proposed. First, the proposed device became supported through state-of-the-art GPS generation. Hence, the gadget could be able to provide real time vicinity of the transferring bus thru Google Map. Secondly RFID become used to update the statistics on the modern bus prevent, to permit the users review the today's bus prevent area and finally turned into the participatory contributions element. Users were capable o read the news feed for unpredictable on-road issues contributed by different bus users who suggested and updated possible arising issues (injuries, breakdowns, etc.) which have been undetectable by using the gadget itself.

Ding Bing Lin have proposed a cell place estimation and monitoring approach for wireless communique structures. The place estimation is based totally at the differences of downlink signal attenuations, which can be used to decide circles composed with the aid of feasible places. Then the actual area is given by way of the intersection of the circles. The extraordinary advantages of this method are the non-necessity of an acknowledged and correct route loss modelling and the discount of shadowing effect. Furthermore, a cellular monitoring approach through linear optimization using easy genetic set of rules was implement to improve the places estimation. This paper has proposed a cell vicinity estimation and monitoring technique for GSM structures. The estimation is based totally on the variations of signal attenuations, and its blessings consist of the non-necessity of an excellent direction loss modelling and the discount. The proposed scheme changed into implemented to a realistic GSM system. Although it could perform better in rural environment than in city because of shadowing, encouraging area accuracy is received in urban city. Furthermore, the proposed monitoring method, linear optimization using GA, smooth and corrected the place mistakes. The corresponding values after monitoring are decreased. With the assist of virtual map, machine screen can almost verify which path the cellular user locates. 
Civil Engineering and Urban Planning: An International Journal (CiVEJ) Vol.5, No.1, March 2018

Pradnya Battin and Dr. S. V. Markande researched on "Location Based Reminder". The telephone carries built-in GPS receive which gets signal from GPS receiver. The software plays Geo location primarily based on GPS reading to locate present location of the person. The favored locations \& responsibilities restored in the database. If task to be reminded is to be had in the database, after that utility will perform contrast of the vicinity which is recognized with the vicinity related with the preferred machine. If the application user is physically near to the defined vicinity, then reminding alert might be given to the person about the project. For including places for specific responsibilities into the database, the utility uses Google Maps. By selecting up the favored location which is displaying on Google Maps, user can upload the favored mission into the database. This could be used for putting reminder in Location Based reminder utility. It isn't always important for the person to bodily gift on the unique location even as entering the reminder within the software.

Karoly Farkas et.al placed the emphasis on the advent of crowd sensing based public shipping statistics service and a prototype smart town software. The interface of this carrier is called TrafficInfo. It is easy and easy-to-use Android utility which visualizes real-time public delivery facts of the given city on Google Maps. The active updates of shipping schedule facts is based on the computerized stop event detection of public transport cars. TrafficInfo is built upon our Extensible Messaging and Presence Protocol (XMPP) based on totally conversation framework what author designed to facilitate the improvement of crowd assisted clever city programs. The paper introduced shortly this framework, than described TrafficInfo in element collectively with the advanced stop occasion detector. In this paper, delivered XMP based totally communication framework that web signed to facilitate the development of crowd assisted smart metropolis packages. Then this provided crowd sensing based totally actual-time public delivery facts provider, implemented on pinnacle of framework, and its front-cease Android utility, referred to as TrafficInfo, in detail collectively with prevented occasion detector. This detector turned into advanced to robotically detect halt events of public transport cars at the stops. As future work, to broaden TrafficInfo in addition and decorate the unique offerings of all the delivered service stages. Moreover, to recruited a substantial user base and perform field experiments with these real users. Their remarks was crucial to plot the instructions for upgrades.

P. Flisberg considered the overall problem of establishing a weighting of many objectives in the riding behavior so that use minimum cost direction generator. This work proposed a solution approach this is based totally on tiers. In the primary stage, researcher identified a set of key routes which are agreed to be the best routes by way of the events. These are measured in detail. In the second one stage, researcher clear up an inverse optimization problem, which ends within the weight parameters for the objective function. With these parameters, it could remedy minimum cost (weighted shortest route) course problems and attained routes that are as close as possible as the desired key routes. The method advised to find values of such weights for the functions. The optimization model to find weights is an inverse shortest path problem formulated in a mixed integer programming model. The method is examined for the Swedish Forestry National Road database. Since2010, it's been in everyday use to set up distances and is to be had for all forestry corporations and haulers in Sweden via a web gadget. Our hassle is to locate weights for the street attributes such that when we solve a MC course trouble between a pair of nodes, we get the corresponding key routes. This hassle is categorized as an inverse problem. Given a (combinatorial) optimization problem and a viable answer, the corresponding inverse optimization hassle is to discover a minimum adjustment of the fee characteristic such that the given answer (key routes) is most reliable solution. In our case, the hassle is a MC problem 
(solved as a weighted shortest course trouble), and the adjustment of the value function (arc costs) is carried out through the weights of the functions.

Sean T. Fursdan and Christine n. Madden discussed the techniques used to evaluate the app's overall performance and resulting evaluation of the range accuracy. The app's stationary variety accuracy was observed to be 4.2 meters (thirteen. 8 toes) and the cellular-to-mobile variety accuracy become located to be 95.7 meters (314 feet) for speeds up to $70 \mathrm{mph}$ and tiers up to twenty miles apart. Additionally, resources of accuracy errors are discussed and quantified. Based upon this facts, a worst-case accuracy prediction equation furnished for instances in which take a look at conditions are known. This equation changed into located to properly expect an accuracy that turned into worst case whilst in comparison to real statistics sets received at some stage in trying out. The RTRT app become developed using the Android Software Development Kit (SDK) furnished with the aid of Google. The SDK presented severe an integrated features to get admission to numerous sensors and abilities of a cell smart device. The RTRT app became designed to perform on cell gadgets jogging Android Application Program Interface (API) variations 10 (Gingerbread) and higher. The app obtained location statistics from the cell device's built-in sensors and transmitted this region facts over a community (mobile or Wireless Fidelity (Wi-Fi) to a second cellular device. The user has the option of choosing Global Positioning System (GPS) or fused records for monitoring the devices vicinity. Implemented via the Google Services API, the fused vicinity service affords the app with the nice available location facts from to be had vicinity providers, along with Wi-Fi and GPS. Once records from the second one device received, the RTRT app method displays the modern vicinity and variety records on the display to the person. The place recorded both gadgets and the variety facts stored in a Structured Query Language (SQL) Lite database and outputted as Comma Separate Variable (CSV) and Keyhole Mark-up Language (KML) documents at test completion. Details regarding the RTRT app necessities, coding, and inner logic had been Documented Architecture of android app based on application fundamentals, developing android application, application framework and android runtimes. Android packages have been written in Java programming language. However it's far crucial to take into account that without the usage of the usual Java Virtual Machine (JVM) android programs cannot be performed. Instead, Google has created a custom VM known as Dalvik. The Android SDK offers an intensive set of software programming interfaces (APIs) that is each contemporary and resistant. Android handset core gadget services are reveal and approachable to all applications. When given the appropriate permissions, Android utility can percentage data among one another and access shared sources at the gadget securely.

Rene Hense et.al described Smart CampusAAU - an open, extendable platform that helped the smooth creation of indoor area based structures. SmartCampusAAU given an app and backend that can be used to permit indoor positioning and navigation in any building. The SmartCampusAAU app available on all essential mobile systems (Android, iPhone and Windows Phone) and helps each tool- and infrastructure-primarily based positioning. SmartCampusAAU additionally given a publicly to be had Data back end that lets in researchers to percentage radio map and vicinity monitoring records. In this paper provided the SmartCampusAAU software platform designed to facilitate indoor positioning and navigation. SmartCampusAAU overcomes the limitations of current offerings via providing help for ubiquitous positioning and navigation on all predominant mobile structures. SmartCampusAAU predicated on crowdsourcing to construct indoor radio maps and graphs and insurance therefore probably limitless. In addition to being on all foremost mobile platforms, the other most important characteristic that made SmartCampusAAU precise that it makes possible for researchers to share radio map- and area tracking facts. This opens up further studies opportunities, now not most effective within region 
Civil Engineering and Urban Planning: An International Journal (CiVEJ) Vol.5, No.1, March 2018

fingerprinting and indoor information control, but indeed anywhere, where get admission to indoor vicinity information is needed. The steps worried in facilitating indoor positioning and navigation in a building are as follows:

1) Add a building and constructing floors

2) Build a radio map to permit indoor positioning

3) Supply symbolic facts for places.

4) Build a graph to permit indoor navigation

$\mathrm{Li} \mathrm{Li}$ included the Android SDK a device emulator, a debugger, libraries exposing functionalities of the Android platform as nicely as numerous code examples and documentation. Maintenance of the Android SDK going hand in hand with the general Android platform improvement. Thus, the SDK library classes evolved to assist maximum latest gadgets however might also include older variations of the Android platform for testing apps on older gadgets. This paper worked on the evolution of inaccessible APIs of the Android framework base. At first, the importance of the phenomenon of inaccessible APIs checked out, wherein discovered that inaccessible APIs continuously applied inside the Android framework, and there honestly no assure of ahead compatibility while the usage of them. Besides, maximum inaccessible APIs may be eliminated in some model updates. Second, at the ability impact of the usage of inaccessible APIs discovered that comparing to available APIs, inaccessible APIs simplest get admission to a specific set of functions of sources and greater risky in addition to much more likely to be removed at the release of a brand new API stage, as opposed to be made publicly in the collect SDK development library. Finally, at the adoption of inaccessible APIs by using 1/3-celebration apps. Experimental outcomes show that there are many apps which can be certainly accessing inaccessible APIs and the utilization are quite specific between malicious and benign apps and additionally observed that some apps leverage a framework called Exposed to ease their works of getting access to inaccessible APIs. Besides, it appears that evidently builders were not seem to taken under consideration the dangers of gets rid of inaccessible APIs, however as a substitute, they might be inquisitive about harnessing immediately the potential of inaccessible APIs. Last but now not the least, not like Apple store, the Google play shop does not have a vetting machine for using inaccessible API strategies.

Manli and Najme Zehra Naqvi proposed a clever public delivery system based on GSM-GPS methodology to song the bus region based totally on which the Arrival times and postpone instances of buses approaching a bus stop are displayed on bus stops to tell passengers so that they could recognize approximately the reputation in their buses and their mode of transport for that reason. In addition, the flaws on this device and improved the gadget in order that it turned out to be less complex, value powerful. The foremost intention depicted the time of arrival of buses at every bus forestall. There were 3 extraordinary modules: Transmitter module, receiver module and a clever phone. The transmitter module equipped with the bus and the receiver module on the bus terminus. GPS module in bus transmitted the latitude and longitude coordinates to the bus terminus via GSM, wherein the arrival and delay times calculated through using these coordinates. The timings supplied to customers via clever phones and displayed on bus terminus. Hence, this system will substantially lessen the tension of commuters. The ATDT algorithm used. 
Civil Engineering and Urban Planning: An International Journal (CiVEJ) Vol.5, No.1, March 2018

ATDT ALGORITHM WOKING: Two variables for preliminary coordinates and new coordinates are initialized. When the modern-day coordinates have received from the INSIDE BUS module, the brand new coordinates assigned with the modern coordinates. A circumstance used to check if the bus is shifting or now not. The previous coordinates are checked towards the new ones. Depending in this condition a selection may be taken if the bus is moving or no longer. If the condition i.e. new coordinates and the initial coordinates are special, then the bus is transferring. Accordingly the longitude and latitude values used to calculate the distance of the bus from the bus forestall and the envisioned time of arrival. This facts displayed on the screen at the bus prevented for the passengers looking forward to the bus. It is likewise sent to the server for the passengers who have the clever phones to get admission to this records from any region.

Nur Rokhman and Lubab Saifuddin used hobby reminder machine based totally on vicinity and time has been proposed. The reminder system makes use of the venue on Foursquare and Google map. By the usage of this reminder system, a notification can be given to the consumer when the consumer's location detected either near the venue or when the time in closed to the time of the hobby. Based at the design, implementation, and testing of the machine, it that may be concluded that:

1. A reminder gadget which works primarily based on the venue region and interest time has been absolutely advanced. The system became built by way of the usage of the APIs of Foursquare and Google Maps. This machine extensively utilized spatial data from Google Maps to explain the place of the authentic venue in geographical coordinates.

2. Based on the checking out results, the device turned into able to notify the user whilst the person changed into in the radius parameters of each venue location. The devices applied notifications while observe changed into detected coming near the closing date.

3. A word of crucial activity is probably alarmed primarily based at the pastime area and the pastime time.

The reminder gadget stored the information which consist the pastime title, the interest contents, the venue name, the venue place, the category, the distance, the geographical coordinates, and the time restriction. Distance become used as a parameter to the reminder gadget. All facts become saved in the form of a string into the SQLite Database. SQLite Database is an Android database device. As a consumer saved an activity information, the utility checked whether or not it turned into an exchange from an old pastime facts or a new interest.

Bhuvana Sekar and Jiang B. Liu described a TriTHEApp Android software, designed to function a vicinity based time saving and protection ensuring software. In other phrases TriTHEApp area primarily based carrier with three capabilities:

1) Tool to announce arrival.

2) Handset robbery monitoring and

3) Emergency call characteristic included in it. 
Tool to announced arrival service designed to make client's uncertain ready time recognized, handset theft tracking designed to tune person's android device, and emergency name feature designed to ensure non-public safety of the android device user. Development of this application led us to pick out few usability issues and to suggest answers to solve those concerns. The tools used for growing this software Eclipse IDE with ADT (Android Developer Tools) plug-in and Android SDK (Software Development Kit).This application prototype is advanced at API degree 16(Android Jellybean 4.1). The cause in the back of choosing this API level is that Android Jellybean OS launch has installed itself because the dominant model of Android that accounting for $40 \%$ market-proportion on android devices and it gives a number of characteristic upgrades. To address this problem, the TriTHEApp utility we evolved includes a 'Tool to announce arrival' characteristic, which modified implementation of GPS tracing to useful resource the customers to realize their absolute wait time/wait time closer to absolute, by using tracking the provider issuer (person with whom the appointment has been made) and calculating the time required for them to attain the meeting place based on their modern-day location

Xianhua Shu et.al introduced the structure and factor fashions of Android, and analysed the anatomy of an Android application such as the functions of Activity, Intent Receiver, Service, Content Provider, and etc. Based on Android, the design method of a location-based cell carrier then supplied. The layout example suggested that it was so smooth to implement self-vicinity, to draw the using trace, to perform question and to flexibly manipulate the actual-time map on Android. The function area based totally on provider which emphasized on Android platform. One can combined a completely zoom and drag enabled map by using adding simply few traces in the java code and XML code of the Android-Default-Application. Through the research on Android architecture and application improvement, and from the layout approach and effects of an software example on this paper, the availability and overall performance of the platform is tested and the design end result also shows the easiness to put into effect self-place, to draw the using trace, to perform queries and to flexibly control the real-time map on Android. The real device also achieved high going for walks performance. The destiny paintings is to lay out a more effective cell place-primarily based device featured with more unique custom designed capabilities based on Android. . It takes some time to trade the records available on Google server.

Ning Sun et.al researched on the conventional Dijkstra algorithms improvements and considers the capabilities of routes: path rankings, constrained pace lane wide variety and so forth. Through the set different factors weight judgment matrix and considering load capability, but the main purposed to design a fast, realistic, consumer-friendly course planning application with load capability and restricted making plans numbers. In the general "directed graph", simplest taken into account the connectivity, and within the path making plans best recollect the connectivity of nodes. So node - arc segment could meet the necessities. During the path network, overlapping routes in path making plans reduced the traffic capability. When use the route planning in navigation systems, customers came to ability users. When measured the potential of a section, those capacity users ought to be introduced to concerns. In order to test the effect of the algorithm, layout and build a fixed of road complete facts tracking and response machine. A realtime tracking the site visitor waft, avenue data associated with the clever equipment and associated facts gathered. The device will use municipal department set up on the street isolation guardrail transformation installation. Among sensor nodes with the aid of ZigBee, Wi-Fi communication protocol consisting of a multi hop community, through the node to the notion of the item to be measured information and could perceive the statistics dispatched to the nearby sink node, implementation of the provisions of the place records series. The nearby sink node is 
Civil Engineering and Urban Planning: An International Journal (CiVEJ) Vol.5, No.1, March 2018

transmitted to the statistics centre through the lengthy distance wireless communication way, consequently it could reach the complete statistics of the complete metropolis. Using the road records of these actual time updates, combining with the statistics of city digital site visitors map system, the routes making plans algorithm is used to make the optimization of avenue planning. System records statistics, evaluation results can be accessed via the App and Web.

\section{TECHNICAL BACKGROUND}

\subsection{Global Positioning System}

The Global Positioning System (GPS) is a utility that gives clients situating, route, and timing administrations. This framework comprises of three sections: the space section, the control portion, and the client fragment. The space section comprises of an ostensible heavenly body of 24 working satellites that transmit one-way flags that give the current GPS satellite position and time. The Control Segment tracks the GPS satellites, transfers refreshed navigational information, and keeps up wellbeing and status of the satellite group of stars. The client portion comprises of the GPS collector hardware and employments the transmitted data to compute the client's three dimensional position and time.

\subsection{Android}

Android is a versatile working framework which offers a bound together way to deal with application improvement. Engineers require to create applications utilizing Android and these applications can keep running on various diverse gadgets, as long as the gadgets are controlled utilizing Android

\subsection{Google Maps}

Google Maps gives a guide of an open Application Programming Interface (Google Maps API). Designers can effortlessly connect the Google Maps administration to their application.

\subsection{Web Client}

The data in the vault can be overseen and seen utilizing the web customer. The client gets the area data from the web customer on their mobiles.

\subsection{Map Service}

The guide benefit is a specialist based which gives both the portable and the web customer with outline. The guide benefit utilizes GPS to track the position of companions or relatives. The area data is refreshed to web customer each time by the cell phone.

\subsection{Data Sharing Centre}

Sharing module deals with images, documents, details of users and location of users. 
Civil Engineering and Urban Planning: An International Journal (CiVEJ) Vol.5, No.1, March 2018

\subsection{PHP}

PHP used to manage database, dynamic content, ST (Session Tracking), even build entire ecommerce sites and it is compiled with various databases. Server side scripting language means PHP and it is embedded with HTML.

\subsection{My SQL}

My SQL works on various OS (Operating System) along with many languages including PHP, C, $\mathrm{C}++, \mathrm{JAVA}$, etc. My SQL is fast, easy to use and works with large datasets as well.

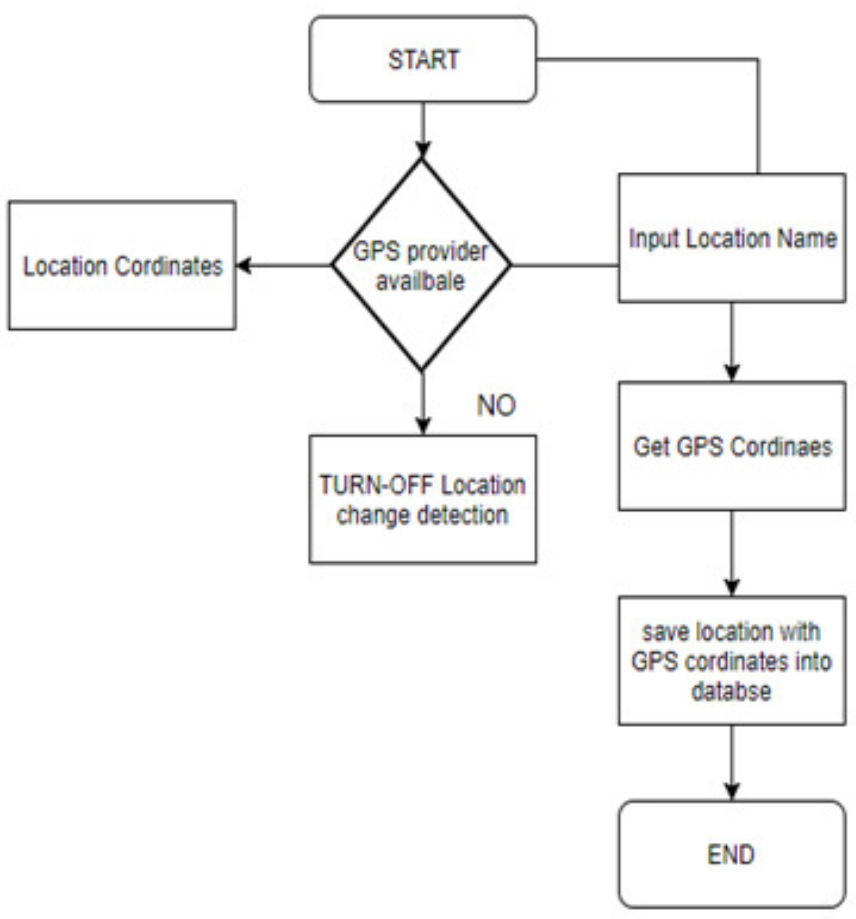

Fig 3.1. Architecture Proposed Framework

\section{Proposed Architecture}

The proposed architecture for location based android mobile app shown the number of technical aspects have been compiled in research. The proposed architecture to developed location based android mobile app as given below:

- $\quad$ Mobile Client

- Web Client

- $\quad$ Map Service

- Data Sharing Centre 


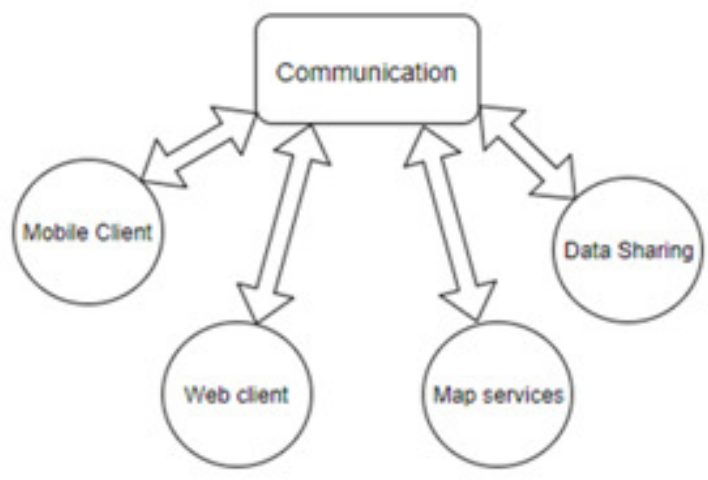

Fig.4.1. Communication system between clients

Global positioning system used to take a longitude and latitude point of area within an accuracy range. Android based mobile app for location tracking leads to find out actual position of person. The following icon are given in app and it compiled below as a serial number -

a) Check GPS connection

b) Make a Profile

c) Capture Photograph

d) Attributes input

e) Save the data

f) Send the data

The steps to be followed for login/Register to Bhuvan Geotag House for Route Planning app are as follows -

Step 1) - Check for GPS accuracy connection/notification on the top. When the accuracy is less than 10m, tap the GPS icon to collect the location (Latitude and Longitude) details. A confirmation window pops-up with GPS accuracy. Tap "OK" if the accuracy is acceptable, otherwise tap "Cancel" to cancel the collection location data and wait for some more time to get better GPS accuracy. Ensure that there is a clear sky view for receiving GPS satellite signals and user standing over the centre of object/structure.

Step 2) - The app has provision to capture and upload two photographs of the location. Tap the "Photo" icon to activate mobile camera for taking photographs. Accept the first photo taken with your mobile camera (Generally front view of structure or event) and tap the "Photo" icon again to take the second photograph. Then accept the second photograph.

Step 3) - The user is expected to upload attribute information about the location by tapping "Attribute" icon. The information such as Project Address or any other related description about the location can be uploaded using this option. 
Civil Engineering and Urban Planning: An International Journal (CiVEJ) Vol.5, No.1, March 2018

Step 4) - Once the user is satisfied with the information collected and ready for uploading the information to Bhuvan server, user may tap the "Send" icon. This will upload all the information collected by the user. User is advised to ensure that mobile data is switched on before tapping the "Send" icon. A confirmation message "Data sent successfully" appears once the upload is successful.

Step 5) - In case of non-availability of internet connectivity, the user is advised to tap "Save" icon. This will store the information collected as a data in the mobile itself. Once the internet connectivity is available, user may follow step 6 to upload the data to Bhuvan server.

Step 6) - In order to upload the saved data to Bhuvan server, user is advised to tap "Manage" icon. Then tap "Send later" icon. This will list the data collected and stored in the mobile. Please select the data that needs to be uploaded and then tap "Send" icon to upload the data to Bhuvan server. A confirmation message "Data sent successfully" appears once the upload is successful.

\section{EXPERIMENT}

The experiment of Bhuvan Geotag House Route Planning shows following window after tap on the installed app. At the starting it will be load first to achieve the latitude and longitude of particular area where user will present. This app designed to people location data by fetching GPS latitude-longitude. This data would be helpful to do network analysis in ArcGIS software to find shortest route or quickest route for those people. It will result into reducing travel time of people. The integration of GIS (Global Information System) and GPS (Global Positioning System) are called as LBS (Location Based System). Location based system use smart mobile devices to collect information regarding to location for optimization of existing route. Network analysis helps to reduce the constraint of route.

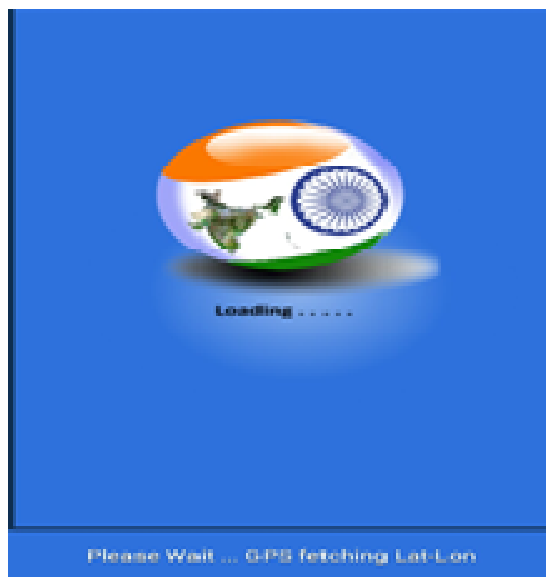

Fig.6.1. Start Screen of Bhuvan Geotag for Route Planning App.

After loading user will get another windows which will consist different icons like GPS, camera, attributes, send and manage, save, exit, profile and help. The user should wait to collect accuracy of GPS more than $10 \mathrm{~m}$. If user will get accuracy below $10 \mathrm{~m}$ then tap on GPS icon to recollect the latitude and longitude point. Once user got longitude and latitude point then user can switch on profile icon for login registration. 
Civil Engineering and Urban Planning: An International Journal (CiVEJ) Vol.5, No.1, March 2018
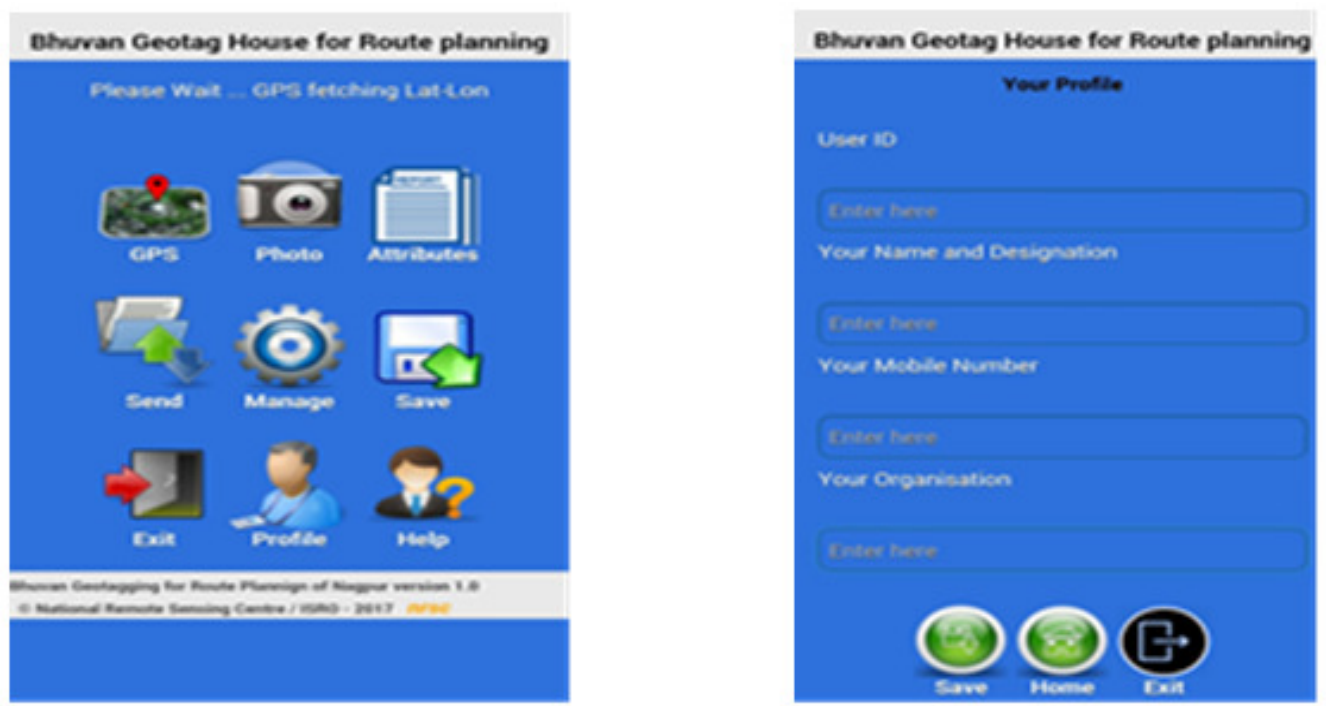

Fig.6.2. Icons Window and Profile Window of App.

Once user have done with profile details then tap on save icon for saving data for further process. Then user can move through home window for giving attributes input details.

In attributes window, attributes input will ask for a personal details about name of user/student, enrolment number, mobile number, transport facility (Require OR not Require), address type (House address location and Pick-up address location). While giving input to the address details care should be taken to select house address location and pick-up address location. If user giving input while going to designation place or organization place the user must choose the pick-up address location at that present time and follow further procedure. If user is going to house or their residential place from organization or designation place then user should choose the house address location after reaching to destination point and follow further procedure Care should be taken to select house address location and pick-up address location.
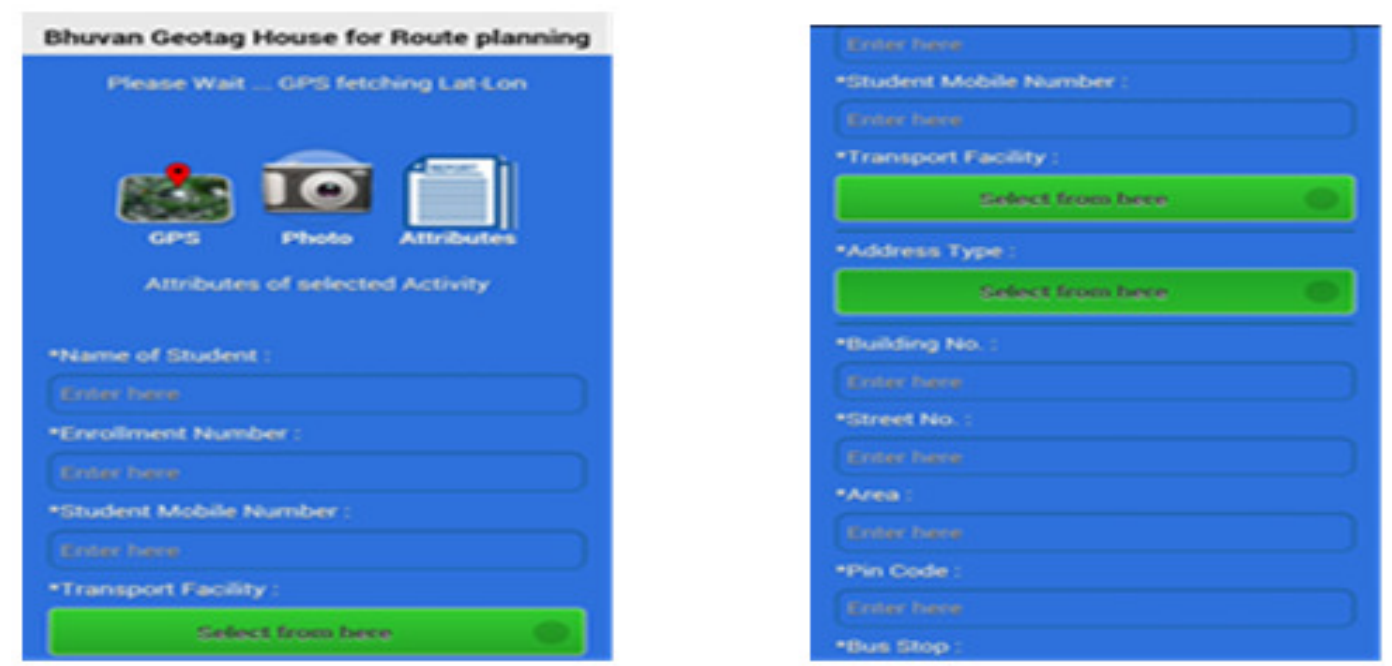

Fig.6.3. Icons Window and Attributes Activity Profile Window of App. 
Civil Engineering and Urban Planning: An International Journal (CiVEJ) Vol.5, No.1, March 2018

If user giving input while going to designation place or organization place the user must choose the pick-up address location at that present time and follow further procedure. If user is going to house or their residential place from organization or designation place then user should choose the house address location after reaching to destination point and follow further procedure.

After filling all these details, user must have to save these data. Data saving will just take a while to save and as the data saved user will get an alert about that data is saved then tap on send icon to share the data with Bhuvan server. These data will be to know starting and destination point of user. To know data about like this it will be helpful to sort out problem of transportation facility of student or employee of organization.

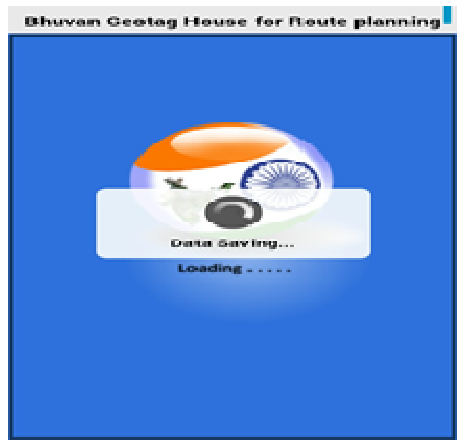

Fig.6.4. Profile Data Saving Window.

Data saving will be done after you have completed with all the user details. Then user can move to manage icon which located on home window of the app. After clicking on manage icon user will get upload data window. If user did not share the data with Bhuvan server may be because of less connectivity of internet then now user can send data by clicking on first string of "upload send later data". Seldom, user uploaded the data but it failed due to some reasons of internet connectivity. So user can able to send that data by clicking on "Upload sent failed data". Once user will have done with all these steps user may see his/her sent data along with number of data have sent. All these steps leads towards the confirmation message of uploaded data.
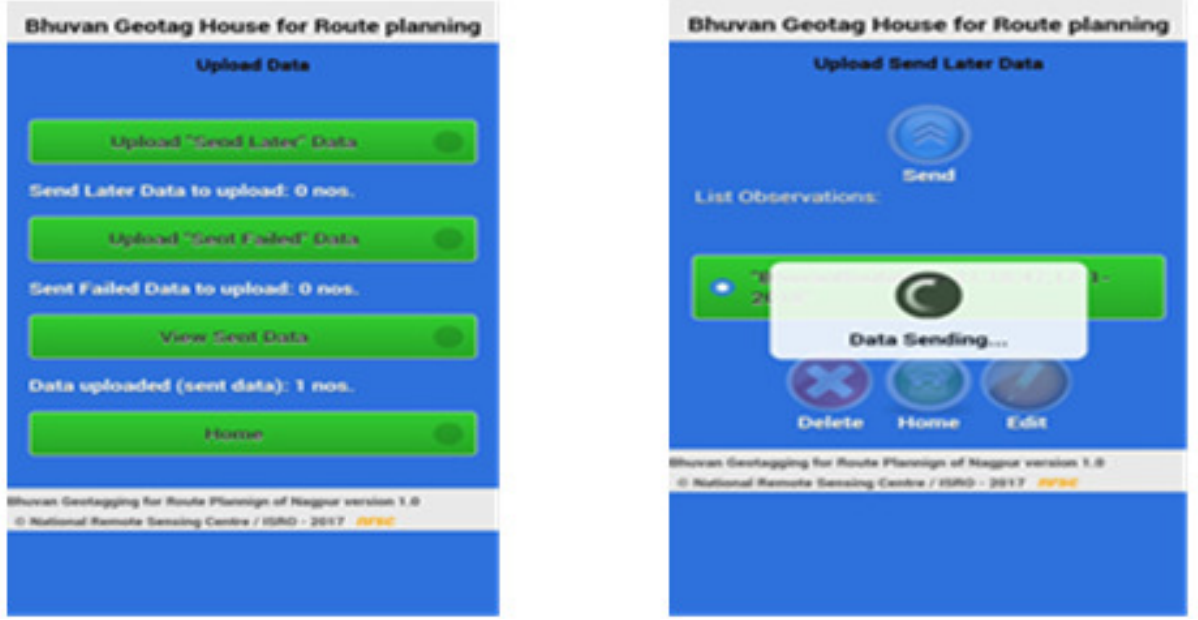

Fig.6.5. Upload Data Window and Data Saving Window. 
As user will get "uploaded data successfully" confirmation message then it will show the direction message as well to get exit of current windows by clicking on "OK" and app will be ready to take another location user.

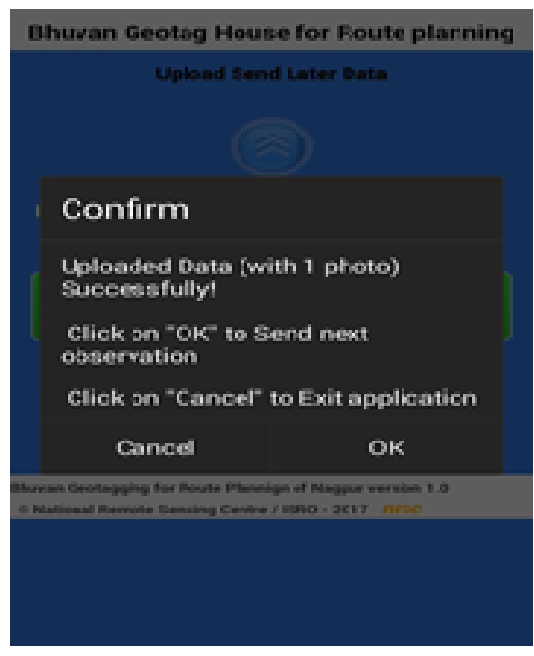

Fig.6.6. Data Successfully Uploaded Confirmation Message

\section{CONCLUSIONS}

From this paper, the work has been done which we can used to track the location of people to sort out problem of congestive traffic route by providing best shortest route to people. Location based service is a pivot contribution to network analysis. This Bhuvan Geotag for Route Planning app can be used for companies, organization and institutional places to find optimal route for employees, workers and students. The actual aim to done this work is to reduce time of travelling, reduce the fuel consumption in travelling and find the best shortest route for people for ease. Now-a-days, people facing the congestion problem in wide range. With increasing population of a country, it is necessary to design the beat optimal route for people. By finding the best optimal route by network analysis will be useful to solve the problem of congestive traffic, consuming excessive time to reach destination point and traffic vehicular activity. Compilation of location based service, Global Positioning System, Network analysis and route planning gives the modified solution over the existing work. Most of people are using smart technology systems for every query about day to day life. The standard of people tries to touch the tangent of smart technologies to get a comfortable life in such case this app for location tracking and network analysis is the biggest acceptable technology to make transportation facility without constraint of time. Due to enhance transportation system according to get a destination point in shortest time and to get a shortest route to reach systems for every query about day to day life. The standard of people tries to touch the tangent of smart technologies to get a comfortable life in such case this app for location tracking and network analysis is the biggest acceptable technology to make transportation facility without constraint of time. Due to enhance transportation system according to get a destination point in shortest time and to get a shortest route to reach. 
Civil Engineering and Urban Planning: An International Journal (CiVEJ) Vol.5, No.1, March 2018

\section{ACKNOWLEDGEMENTS}

I would like to thanks who have supported me for research Dr. Rajashree Bothale (Research Guide) and Dr. P. Y. Pawade (Deparmental Guide).

\section{REFERENCES}

[1] Benjamin Y.O. Low, Samsul Haimi Dahlan, Mohd Helmy Abd Wahab,"Real-time Bus Location and Arrival Information System," Research Center for Applied Electromagnetic (EMCenter), Embedded Computing Research Focus Group, Universiti Tun Hussein Onn Malaysia, 2016 IEEE Conference on Wireless Sensors (ICWiSe).

[2] Ding-Bhg Lin, Rong-Terng Juang, and Hsin-Piao Lin, "Mobile Location Estimation and Trracking for GSM", Institute of Computer and Communication, National Taipei University of Technolog, 2004 IEEE Conference.

[3] Pradnya Battin and Dr. S.D.Markande, "Location Based Reminder Android Application Using Google Maps API, Department of Electronics \& Telecommunication NBN Sinhgad School of Engineering Ambegaon, Pune, 2016 International Conference on Automatic Control and Dynamic Optimization Techniques (ICACDOT) International Institute of Information Technology (IIT), Pune.

[4] Karoly Farkas, Gabor Feher, Andras Benczur, and Csaba Sidl, "Crowdsending Based Public Transport Information Service in Smart Cities," Telecommunications and Informatics, Debrecen, Hungary, and the Budapest University ofTechnology and Economics,Budapest Hungary, August 2015, IEEE Communications Magazine.

[5] P. Flisberg, B. Lidén, M. Rönnqvist, and J. Selander, "Route selection for best distances in road databases based on drivers' and customers'preferences," The Forestry Research Institute of Sweden, SE-75183 Uppsala, Sweden, Can. J. For. Res. 42: 1126-1140 (2012), 3 April 2012, Published by NRC Research Press.

[6] Sean T. Fursdon and Christine M. Madden, "Real Time Range Tracker (RTRT):Range Accuracy Performance," Space and Naval Warfare Systems Center Atlantic North Charleston, SC USA, the U.S. Department of Navy under Section 219 of the Fiscal Year 2009 National Defense Authorization Act, which established the NISE program.

[7] Reetesh V. Golhar, Prasann A. Vyawahare2, Pavan H. Borghare3, and Ashwini Manusmare4, "Design And Implementation Of Android Base Mobile App For An Institute," Dept. of ETRX,DMIETR Wardha, technology to make transportation facility without constraint of time. Due to enhance transportation system according to get a destination point in shortest time and to get a shortest route to reach Maharashtra, 2016 IEEE, International Conference on Electrical, Electronics, and Optimization Techniques (ICEEOT).

[8] Ren'e Hansen, Bent Thomsen, Lone Leth Thomsen, and Filip Stubkjær Adamsen, "SmartCampusAAU - An Open Platform Enabling Indoor Positioning and Navigation," Department of Computer Science, Aalborg University, 2013 IEEE 14th International Conference on Mobile Data Management.

[9] Gil Heo, Genong Yu and Liping Di, "A Reconfigurable Open GeoSMS Mobile Client App Design for Android Smartphones”, Center for Spatial Information Science and Systems (CSISS) George Mason University. 
Civil Engineering and Urban Planning: An International Journal (CiVEJ) Vol.5, No.1, March 2018

[10] Li Li, Tegawend’e F. Bissyand'e, Yves Le Traon and Jacques Klein, "Accessing Inaccessible Android APIs:An Empirical Study," 2016 IEEE International Conference on Software Maintenance and Evolution.

[11] Gil Heo, Genong Yu and Liping Di, "A Reconfigurable Open GeoSMS Mobile Client App Design for Android Smartphones", Center for Spatial Information Science and Systems (CSISS) George Mason University.

[12] Manali, Najme Zehra Naqvi, "Smart Public Transport System Using Mobile phone based sensing," Department of Computer Science and Engineering, Delhi, India, Embedded Computing Research Focus Group, 2015 IEEE INDICON.

[13] Nur Rokhman and Lubab Saifuddin, "Mobile Location Estimation and Trracking for GSM", Department of Computer Sciences and Electronics Gadjah Mada University Yogyakarta, Indonesi, 2016 2nd International Conference on Science in Information Technology (ICSITech).

[14] Bhuvana Sekar and Jiang B.Liu, "Location and Time Based Reminder System on Android Mobile Device, Computer Science \& Information Systems Department Bradley UniversityPeoria, Illin ois, U.S.A, 2014 IEEE.

[15] Xianhua Shu, Zhenjun Du, Rong Chen, "Research on Mobile Location Service Design Based on Android," School of Information Science and Technology Dalian Maritime UniversityDalian, China, 2009 IEEE.

[16] Ning Sun, Guangjie Han, Pengfei Duan and Jiayao Tan, "A Global and Dynamitic Route Planning Application for Smart Transportation," College of Internet of Things Engineering Hohai University Changzhou, China, 2015 First International Conference on Computational Intelligence Theory, Systems and Applications.

[17] Sudhir K. Tiwari, Gaurav K. Varshney, Mohammed A. Qadeer and M. Sarosh Umar, "JustQuick: Intelligent LBS usingAndroid with GPS and Geo-Tagging Applications," Department of Computer Engineering, Zakir hussain college of Engineering and Technology, Aligarh Muslim University,2016,IEEE. 